\title{
Niveles de algebrización de las prácticas matemáticas escolares. Articulación de las perspectivas ontosemiótica y antropológica
}

\author{
Juan D. Godino, Universidad de Granada (España) \\ Teresa Neto, Centro de Investigación "Didáctica y Tecnología en la Formación de \\ Profesores", CIDTFF, Universidad de Aveiro (Portugal) \\ Miguel R. Wilhelmi, Universidad Pública de Navarra (España) \\ Lilia Aké, Universidad de Colima (México) \\ Silvia Etchegaray, Universidad Nacional de Río Cuarto (Argentina) \\ Aitzol Lasa, Universidad Pública de Navarra (España)
}

Recibido el 31 de mayo de 2015; aceptado el 2 de septiembre de 2015

\begin{abstract}
Niveles de algebrización de las prácticas matemáticas escolares. Articulación de las perspectivas ontosemiótica y antropológica.

\section{Resumen}

En el marco del enfoque ontosemiótico del conocimiento y la instrucción matemáticos se ha propuesto una caracterización del razonamiento algebraico en Educación Primaria basada en la distinción de tres niveles de algebrización. Tales niveles se definen teniendo en cuenta los tipos de representaciones usadas, los procesos de generalización implicados y el cálculo analítico que se pone en juego en la actividad matemática correspondiente. En este trabajo ampliamos el modelo anterior mediante la inclusión de otros tres niveles más avanzados de razonamiento algebraico que permiten analizar la actividad matemática en Educación Secundaria. Estos niveles están basados en la consideración de 1) el uso y tratamiento de parámetros para representar familias de ecuaciones y funciones; 2) estudio de las estructuras algebraicas en si mismas, sus definiciones y propiedades. Asimismo, se analizan las concordancias y complementariedades de este modelo con las tres etapas del proceso de algebrización propuestas en el marco de la teoría antropológica de lo didáctico.
\end{abstract}

Palabras claves. Razonamiento algebraico, Educación Primaria, Educación Secundaria, enfoque ontosemiótico, teoría antropológica, formación de profesores

Algebrization levels of school mathematics practices. Networking of the Onto-semiotic and Anthropological perspectives

Abstract

Para citar: Godino, J.D., Neto, T. Wilhelmi, M.R., Aké, L., Etchegaray, S. \& Lasa, A. (2015). Niveles de algebrización de las prácticas matemáticas escolares. Articulación de las perspectivas ontosemiótica y antropológica. Avances de Investigación en Educación Matemática, 8, 117 - 142. 
Niveles de algebrización de las prácticas matemáticas escolares. Articulación de las perspectivas ontosemiótica y antropológica.

Based on the onto-semiotic approach to mathematical knowledge and instruction a characterization of algebraic reasoning in primary education has been proposed, distinguishing three levels of algebraization. These levels are defined taking into account the types of representations used, generalization processes involved and the analytical calculation at stakes in mathematical activity. In this paper we extend this previous model by including three more advanced levels of algebraic reasoning that allow to analyze mathematical activity carried out in secondary education. These new levels are based on the consideration of 1) using and processing parameters to represent families of equations and functions; 2) the study of algebraic structures themselves, their definitions and properties. Furthermore, concordances and complementarities of this model with the three stages of algebrization proposed under the anthropological theory of didactics are analyzed.

Key words. Algebraic reasoning, primary education, secondary education, onto-semiotic approach, teachers' education

Níveis de algebrização das práticas matemáticas escolares. Articulação das perspectivas Ontossemiótica e Antropológica

\section{Resumo}

No enfoque ontossemiótico do conhecimento e do ensino e aprendizagem da matemática propõe-se uma caraterização do pensamento algébrico no Ensino Básico baseada na distinção de três níveis de algebrização. Tais níveis definem-se tendo em conta os tipos de representações utilizadas, os processos de generalização implicados e o cálculo analítico que se põe em jogo na atividade matemática correspondente. Neste trabalho ampliamos o modelo anterior mediante a consideração de outros três níveis mais avançados do pensamento algébrico que permitem analisar a atividade matemática ao nível do Ensino Secundário. Estes níveis baseiam-se no seguinte: 1) uso e tratamento de parâmetros para representar familias de equações e funções; 2) estudo das estruturas algébricas em si mesmas, suas definições e propriedades. Além disso, analisam-se as concordâncias e complementaridades deste modelo com as três etapas do processo de algebrização propostas na teoria antropológica do didático.

Palavras-chave. Pensamento algébrico, Ensino Básico, Ensino Secundário, enfoque ontossemiótico, teoria antropológica, formação de professores

Niveaux d'algébrisation des pratiques mathématiques scolaires. Articulation des approches onto-sémiotique et anthropologique

\section{Résumé}

Dans le cadre de l'approche onto-sémiotique de la connaissance et de l'enseignement mathématiques, on propose pour l'École Élémentaire une caractérisation du raisonnement algébrique structurée en trois niveaux d'algébrisation. On caractérise ces niveaux d'algébrisation selon les représentations utilisées, les processus de généralisation impliqués et le calcul analytique mis en jeux à l'activité mathématique. Dans cet article, ce modèle de raisonnement algébrique élémentaire est étendu pour pouvoir analyser l'activité mathématique dans l'Enseignement Secondaire. On propose trois niveaux additionnels basés sur : 1) l'utilisation et le traitement des paramètres pour la représentation des familles des équations et des fonctions; 2) l'étude des structures algébriques eux-mêmes, leurs définitions et propriétés. De même, on analyse les concordances et les complémentarités de ce modèle avec les trois étapes de processus d'algébrisation proposées par la théorie anthropologique du didactique.

Mots-clefs : Raisonnement algébrique, Enseignement Élémentaire, Enseignement Secondaire, Approche onto-sémiotique, Théorie anthropologique du didactique, Formation des enseignants.

\section{Introducción}

La identificación de los rasgos característicos del razonamiento algebraico es un tema que ha atraído la atención de diversos investigadores en el campo de la educación matemática, ya que es necesaria para promover dicho razonamiento en los distintos niveles de Educación Primaria y Secundaria (Chevallard \& Bosch, 2012; Filloy, Puig \& Rojano, 2008; Kieran, 2007; Ruiz-Munzón, Bosch \& Gascón, 2011). Dependiendo de cómo se conciba el álgebra escolar se tomarán decisiones sobre su introducción 
temprana desde los primeros niveles educativos, o se retrasará hasta la Educación Secundaria; asimismo podrán variar las estrategias instruccionales correspondientes. De hecho, el programa de investigación y desarrollo en que se basa el "early algebra" (Cai \& Knuth, 2011; Carraher \& Schliemann, 2007), está apoyado en una concepción del álgebra que reconoce indicios de pensamiento algebraico en actividades matemáticas asumibles desde los primeros niveles educativos (NCTM,2000).

Existen excelentes visiones de conjunto (surveys) que sintetizan la gran cantidad de investigaciones sobre los distintos enfoques y facetas implicadas en la enseñanza y aprendizaje del álgebra en el sistema escolar (facetas epistemológica, cognitiva y de enseñanza). Socas (2011) realiza una síntesis de estudios internacionales referidos al desarrollo del álgebra en la escuela, atendiendo a cuestiones semióticas (lenguaje y representación), mediacionales (recursos tradicionales y tecnológicos), curriculares (inclusión de contenidos novedosos), etc. Concluye que uno de los retos fundamentales actuales sigue siendo la caracterización del pensamiento algebraico.

¿Qué es el pensamiento algebraico y cuáles son las razones esenciales de la actividad algebraica que deben constituir las metas que tenemos para el aprendizaje de los alumnos en este campo? Esta caracterización del Pensamiento algebraico permitirá señalar con claridad las metas para la educación de los alumnos en cada etapa educativa: "Early Algebra”, Prealgebra, Álgebra en la Educación Secundaria Obligatoria (Socas, 2011, p. 26)

Así, aunque se ha avanzado mucho en la caracterización del álgebra escolar, el problema no está completamente resuelto, particularmente en lo que se refiere a la conexión entre el álgebra en Primaria y Secundaria. Este será pues el objetivo esencial del trabajo.

En trabajos previos (Aké, Godino, Gonzato \& Wilhelmi, 2013; Godino, Aké, Gonzato \& Wilhelmi, 2014) hemos propuesto un modelo de pensamiento algebraico para la Educación Primaria en el que distinguimos tres niveles de razonamiento algebraico, estableciendo además criterios para identificar la actividad matemática puramente aritmética (nivel 0 de algebrización) y distinguirla de los progresivos niveles de algebrización. A la actividad claramente algebrizada se asigna un nivel 3 y se establecen otros dos niveles intermedios de actividad proto-algebraica.

Los criterios para delimitar los distintos niveles están basados en el tipo de objetos y procesos matemáticos implicados en la actividad matemática, de acuerdo con el Enfoque Ontosemiótico (EOS) del conocimiento y la instrucción matemáticos (Godino, Batanero \& Font, 2007; Godino, 2012). Los niveles de algebrización se asignan a la actividad matemática que realiza el sujeto que resuelve un problema o tarea matemática, no a las propias tareas, las cuales se pueden resolver de distintas maneras, pudiendo poner en juego una actividad algebraica diferente. Ello no quiere decir que no haya tareas intrínsecamente algebraicas; esto ocurre cuando en el propio enunciado de la tarea se ponen en juego objetos algebraicos (incógnitas, ecuaciones, variables, parámetros, funciones o estructuras algebraicas). No obstante, incluso en el caso de que una tarea esté formulada "algebraicamente" su resolución podría ser categorizada como aritmética. Por ejemplo, la tarea, "Resuelve la ecuación: $x^{2}-5 x+6=0$ ", podría ser resuelta por un estudiante dando valores a la incógnita:

Si $x=0$, la expresión toma el valor 6 ; si $x=1,2$; si $x=2,0$, luego es solución; si $x=3$ : 0 , luego también es solución.

El proceso podría también estructurarse mediante una tabla, dando valores de manera sistemática y extrayendo información adicional relativa al comportamiento de la parábola asociada (tabla 1). 
Niveles de algebrización de las prácticas matemáticas escolares. Articulación de las perspectivas ontosemiótica y antropológica.

Tabla 1. Resolución tabular de la ecuación $x^{2}-5 x+6=0$

\begin{tabular}{|c|c|c|c|c|c|c|c|c|c|c|c|}
\hline$x$ & $\ldots$ & -4 & -3 & -2 & -1 & 0 & 1 & 2 & 3 & 4 & $\ldots$ \\
\hline$y=x^{2}-5 x+6$ & $\ldots$ & 42 & 30 & 20 & 12 & 6 & 2 & 0 & 0 & 2 & $\ldots$ \\
\hline
\end{tabular}

De hecho, estos procedimientos numéricos no representan únicamente un estado previo a la resolución algebraica mediante la fórmula general de cálculo de las raíces de las ecuaciones de $2^{\circ}$ grado; es preciso mostrar sus limitaciones y potencialidades:

a) Limitaciones. Hay ecuaciones que no tienen solución entera (por ejemplo, $x^{2}+x$ $-1=0$ ) y que, por lo tanto, el procedimiento resultaría más costoso.

b) Potencialidades. El método numérico permite la obtención de soluciones (por defecto y por exceso) de ecuaciones de grado 3 o superior, aportando un procedimiento integrador para ecuaciones resolubles por Ruffini (por ejemplo, $x^{3}+7 x^{2}-4 x-4=0$ ) y otras que no lo son (por ejemplo, $x^{3}+7 x^{2}-4 x-1=0$ ).

En este trabajo se extiende el modelo de los niveles de algebrización a la actividad matemática que se realiza en la Educación Secundaria (ESO y Bachillerato). Esta extensión se apoya también en las distinciones ontosemióticas sugeridas por el EOS, particularmente en la presencia, uso y tratamiento de parámetros, tanto en actividades de tipo estructural como funcional. Así, en la sección 2, se resumen los rasgos de los niveles de razonamiento algebraico en Educación Primaria. En la sección 3, se definen los tres nuevos niveles de algebrización para la Educación Secundaria, indicando algunos ejemplos ilustrativos y la conexión de los niveles con la presencia de discontinuidades ontosemióticas. En la sección 4, se propone una síntesis de las tres etapas del proceso de algebrización propuestas en el marco de la Teoría Antropológica de lo Didáctico (TAD) (Chevallard, 1992; 1999) y se compara este modelo con el desarrollado en el marco del EOS. Finalmente, el trabajo concluye con una síntesis, algunas implicaciones para la formación de docentes y cuestiones abiertas.

\section{Niveles de razonamiento algebraico en Educación Primaria}

En la Tabla 2, se resumen las características esenciales de los tres niveles de algebrización descritos en Godino et al. (2014), junto con el nivel 0 (ausencia de rasgos algebraicos). En esta tabla, se aporta asimismo un ejemplo que ayuda a la comprensión de los niveles, los cuales están basados en las siguientes distinciones ontosemióticas:

1) La presencia de "objetos algebraicos" intensivos de un segundo grado de generalidad $^{1}$.

2) El tratamiento que se aplica a dichos objetos (operaciones, transformaciones basadas en la aplicación de propiedades de las estructuras algebraicas correspondientes).

1 Considerando que los números naturales son también objetos intensivos (entidades generales, abstractas) que emergen de colecciones de objetos perceptibles y de las acciones que se realizan con ellos, es necesario atribuirles un primer grado de generalidad o intensión. En el nivel 0 de algebrización no se puede decir que no intervengan objetos intensivos, sino que a tales objetos corresponde un primer grado de intensión. La atribución de un carácter algebraico a una práctica matemática supone la intervención de intensivos al menos de un segundo grado de generalización, es decir, clases de intensivos de grado 1. 
3) Tipos de lenguajes usados (natural, icónico, gestual o simbólico).

El fundamento para definir los distintos niveles de algebrización es de índole ontosemiótica, esto es, se tiene en cuenta la diversa naturaleza de los objetos y procesos matemáticos que intervienen en las prácticas operativas y discursivas que realiza un sujeto epistémico o ideal (punto de vista institucional).

Tabla 2. Rasgos característicos de los niveles de razonamiento algebraico elemental

Tarea: Para ir a la escuela los alumnos utilizan dos medios de locomoción. Por cada alumno que va en coche hay 3 que van andando. Si hay 212 alumnos en la escuela, ¿Cuántos alumnos utilizan cada medio de locomoción?

\begin{tabular}{|c|c|c|c|}
\hline NIVELES & OBJETOS & TRANSFORMACIONES & LENGUAJES \\
\hline \multirow{2}{*}{0} & $\begin{array}{l}\text { Intervienen objetos } \\
\text { intensivos de primer } \\
\text { grado. En tareas } \\
\text { estructurales pueden } \\
\text { intervenir datos } \\
\text { desconocidos. }\end{array}$ & $\begin{array}{l}\text { Se opera con objetos intensivos de } \\
\text { primer grado (números particulares). }\end{array}$ & $\begin{array}{l}\text { Natural, numérico, } \\
\text { icónico, gestual; pueden } \\
\text { intervenir símbolos que } \\
\text { refieren a objetos } \\
\text { extensivos o datos } \\
\text { desconocidos }\end{array}$ \\
\hline & \multicolumn{3}{|c|}{$\begin{array}{l}\text { Ejemplo de resolución: } \\
\text { Si de cada } 3 \text { alumnos que van andando hay } 1 \text { que va en coche, de cada } 4 \text { alumnos en total } \\
(3+1) \text { hay } 1 \text { que va andando (la cuarta parte), por lo tanto de cada } 200 \text { alumnos, } 50 \text { irían en } \\
\text { coche (la cuarta parte); de cada } 12 \text { alumnos } 3 \text { irían en coche. Por tanto, } 53 \text { alumnos irían } \\
\text { en coche. La solución sería } 53 \text { alumnos van en coche mientras que el triple de 53, es decir, } \\
159 \text { van andando. }\end{array}$} \\
\hline \multirow[b]{2}{*}{1} & $\begin{array}{l}\text { Intervienen de manera } \\
\text { implícita objetos } \\
\text { intensivos de grado } 2 \text {, } \\
\text { esto es, clases de } \\
\text { intensivos de grado } 1 .\end{array}$ & $\begin{array}{l}\text { Se aplican relaciones y propiedades } \\
\text { genéricas de las operaciones con } \\
\text { objetos intensivos de primer grado, } \\
\text { tanto en tareas estructurales como } \\
\text { funcionales. }\end{array}$ & $\begin{array}{l}\text { Natural, numérico, } \\
\text { icónico, gestual; pueden } \\
\text { intervenir símbolos que } \\
\text { refieren a los intensivos } \\
\text { intervinientes. }\end{array}$ \\
\hline & \multicolumn{3}{|c|}{$\begin{array}{l}\text { Ejemplo de resolución: } \\
\text { Por cada } 4 \text { alumnos hay } 3 \text { que van andando. Podemos plantear la siguiente } \\
\text { proporcionalidad: } \\
\text { 4(niños) ----> } 3 \text { van andando } \\
212 \text { (niños en la escuela) -----> x van andando } \\
\qquad \frac{4}{3}=\frac{212}{x} ; x=3 \times 212 / 4 \\
\text { Una vez que obtenemos el número de los que van andando a clase, solo queda restar al } \\
\text { número total de alumnos, los que van andando, para obtener los que acuden al colegio en } \\
\text { coche. } 212-159=53 \text { niños van en coche. }\end{array}$} \\
\hline \multirow[t]{2}{*}{2} & $\begin{array}{l}\text { Intervienen } \\
\text { indeterminadas o } \\
\text { variables como } \\
\text { expresión de los } \\
\text { intensivos de grado } 2 \text {. }\end{array}$ & $\begin{array}{l}\text { En tareas estructurales las ecuaciones } \\
\text { son de la forma } A x+B=C \text {. } \\
\text { En tareas funcionales se reconoce la } \\
\text { generalidad pero no se opera con las } \\
\text { variables para obtener formas } \\
\text { canónicas de expresión. }\end{array}$ & $\begin{array}{l}\text { Simbólico - literal, } \\
\text { usado para referir a los } \\
\text { intensivos reconocidos, } \\
\text { aunque ligados a la } \\
\text { información del contexto } \\
\text { espacial y temporal. }\end{array}$ \\
\hline & \multicolumn{3}{|c|}{$\begin{array}{l}\text { Ejemplo: } \\
212=x+3 x \\
212=4 x ; x=212 / 4 ; x=53 \\
53 \text { alumnos van en coche y } 212-53=159 \text { van andando }\end{array}$} \\
\hline \multirow[t]{2}{*}{3} & $\begin{array}{l}\text { Intervienen } \\
\text { indeterminadas, } \\
\text { incógnitas, ecuaciones, } \\
\text { variables y funciones } \\
\text { particulares. }\end{array}$ & $\begin{array}{l}\text { En tareas estructurales las ecuaciones } \\
\text { son de la forma } A x+B=C x+D \text {. } \\
\text { Se opera con las indeterminadas o } \\
\text { variables. }\end{array}$ & $\begin{array}{l}\text { Simbólico - literal; los } \\
\text { símbolos se usan de } \\
\text { manera analítica, sin } \\
\text { referir a la información } \\
\text { del contexto. }\end{array}$ \\
\hline & \multicolumn{3}{|l|}{ Ejemplo: } \\
\hline
\end{tabular}




\begin{tabular}{|c|c|}
\hline & $\begin{array}{l}x=\text { Alumnos que van en coche } \\
y=\text { Alumnos que van andando } \\
\begin{array}{l}x+y=212 ; \quad x+3 x=212 ; \\
y=3 x ; \quad 4 x=212 ; x=212 / 4=53\end{array}\end{array}$ \\
\hline
\end{tabular}

Entre las múltiples combinaciones que sería posible establecer variando los tipos de objetos, transformaciones y lenguajes, lo que es indicativo de la riqueza y variedad del razonamiento algebraico, hemos seleccionado las descritas en la Tabla 2 como criterios para delimitar los niveles 1,2 y 3. Se asume que se incrementa la algebrización a medida que intervienen objetos intensivos de segundo grado (clases o tipos de intensivos de primer grado, como los números naturales), se expresan de manera alfanumérica y se opera con ellos de manera analítica (sintáctica). Se trata, por tanto, de identificar criterios para describir el progresivo desarrollo del objeto intensivo correspondiente, indicando fases en el proceso de reificación del objeto (Sfard y Lichenskin, 1994).

La emergencia del nivel 1 de algebrización ocurre en el primer encuentro con objetos intensivos de grado 2 cuya generalidad se reconoce de manera explícita mediante lenguajes natural, numérico, icónico o gestual. Pueden intervenir símbolos que refieren a los intensivos reconocidos, pero sin operar con dichos objetos. En tareas estructurales se aplican relaciones y propiedades de las operaciones y pueden intervenir datos desconocidos expresados simbólicamente. En tareas funcionales se reconoce la generalidad aunque expresada en un lenguaje diferente al simbólico-literal.

El nivel 2 de algebrización se manifiesta en la intervención de indeterminadas o variables expresadas con lenguaje simbólico - literal para referir a los intensivos de grado 2 reconocidos (p. e., conjuntos de números naturales que cumplen determinadas condiciones), aunque ligados a la información del contexto espacial temporal. En tareas estructurales las ecuaciones son de la forma $A x+B=C(A, B, C \in \mathbf{R})$. En tareas funcionales se reconoce la generalidad, pero no se opera con las variables para obtener formas canónicas de expresión.

El nivel consolidado de algebrización (nivel 3) supone la intervención de objetos intensivos de grado 2 representados de manera simbólica - literal y se opera con ellos; se realizan transformaciones en la forma simbólica de las expresiones conservando la equivalencia. Se realizan tratamientos con las incógnitas para resolver ecuaciones del tipo $A x+B=C x+D \quad(A, B, \quad C, D \in \mathbf{R})$ y la formulación simbólica $\mathrm{y}$ descontextualizada de reglas canónicas de expresión de funciones y patrones. Hemos asociado al nivel 2 el tratamiento de las "ecuaciones aritméticas" (Filloy y Rojano, 1989), esto es, las ecuaciones en las que la incógnita aparece solo en uno de los lados de la ecuación, p. e. $3 x+5=10$, mientras que las ecuaciones en las que la incógnita está en ambos miembros de la igualdad son indicativas de un cambio sustantivo y definen la emergencia del nivel 3.

Sfard y Linchevski (1994) resaltan el papel crucial de la reificación de los conceptos de ecuación y función en la progresión del pensamiento algebraico de los estudiantes, distinguiendo una primera fase en la que dichos conceptos intervienen de manera informal y operacional antes de ser reconocidos como objetos expresados de manera estructural y simbólica.

El álgebra retórica (así es como se llama al álgebra verbal por los historiadores) era practicada desde los primeros tiempos hasta el siglo dieciséis. Este es también el tipo de álgebra encontrada por los niños en la escuela actual bastante antes de que se introduzca cualquier notación formal (Sfard y Linchevski, 1994, p. 197). 
Esta álgebra informal y operacional se corresponde con los niveles 1 y 2 (protoalgebraicos) descritos. Evidentemente, dichos niveles no agotan los procesos de "algebrización", sino que describen el paulatino enriquecimiento de herramientas para la resolución de problemas con un grado creciente de generalización y simbolización y evolucionan hacia niveles superiores al final de la Educación Primaria y primer ciclo de Secundaria.

\section{Niveles de razonamiento algebraico en Educación Secundaria}

El modelo de los niveles de razonamiento algebraico elemental (RAE) que hemos elaborado en trabajos previos (Aké et al., 2013; Godino et al., 2014) permite describir el álgebra escolar, identificando formas de razonamiento algebraico en prácticas operativas y discursivas propias de la Educación Primaria. Esta nueva visión del álgebra precisa su desarrollo en la Educación Secundaria. Así, en este trabajo, articulamos y extendemos el modelo de los niveles de algebrización de la primera etapa educativa con el desarrollo el razonamiento algebraico en secundaria, reconociendo tres niveles de algebrización adicionales propios de esta etapa educativa. Es claro que los niveles 1, 2 y 3 continúan manifestándose en secundaria, en particular, lograr el dominio del nivel 3 suele ser un objetivo central en el primer curso de secundaria.

El uso de parámetros y su tratamiento puede ser un criterio para delimitar niveles superiores de algebrización, ya que está ligado a la presencia de familias de ecuaciones y funciones, y por tanto, implica nuevas "capas" o grados de generalidad (Radford, 2011). Como afirman Caspi y Sfard (2012, p. 47):

Si cada capa en la jerarquía es un discurso sobre su predecesor, una introducción de una nueva capa antes de que el estudiante domine la precedente conlleva el riesgo de que el estudiante simplemente no sabría a qué se refiriere el nuevo discurso.

El primer encuentro con los parámetros lo vamos a ligar a un cuarto nivel de algebrización y la realización de cálculos o tratamientos conjuntos con parámetros y variables a un quinto nivel. El estudio de estructuras algebraicas específicas lleva a reconocer un sexto nivel de algebrización de la actividad matemática.

Drijvers (2003) justifica la importancia de los parámetros en el aprendizaje del álgebra en un contexto computacional por cuatro motivos (pp. 60-62):

1) El uso de parámetros fomenta la reificación de fórmulas y de expresiones algebraicas; asimismo, permite abordar la dualidad proceso-objeto (dificultad central en el aprendizaje del álgebra).

2) El uso de parámetros es un medio de generalización. Los parámetros permiten determinar familias de objetos que pueden ser modelizados y simbolizados mediante una estructura común, hecho que dota de significado a los objetos y procedimientos algebraicos.

3) El uso de parámetros permite a los estudiantes revisar y articular los diferentes usos de la noción de variable.

4) El uso de parámetros permite a los estudiantes desarrollar la comprensión sobre el significado y la estructura de fórmulas algebraicas y expresiones, como una forma de dotar sentido a los símbolos.

Estos usos están condicionados por las distintas funciones contextuales que cumplen los parámetros, como: registro numérico, cantidad cambiante, generalizador o incógnita (Drijvers, 2003, pp. 68-70). La comprensión y articulación flexible por los 
estudiantes de los siguientes usos y funciones contextuales condicionan las trayectorias de aprendizaje del álgebra escolar:

1) El parámetro como registro numérico (placeholder): En esta concepción, uso o significado, el parámetro se ve como una posición, un lugar vacío en el que se pueden insertar valores numéricos y desde el cual se pueden recuperar. El valor en la "caja vacía" es fijo, conocido o desconocido; el foco no está en encontrar el valor desconocido. Gráficamente, al asignar un valor al parámetro se obtiene un gráfico particular. "Este es el nivel básico de comprensión del concepto de parámetro: el parámetro como registro para un valor constante que no cambia" (Drijvers, 2003, p. 68).

2) El parámetro como cantidad cambiante: En esta concepción el parámetro continúa representando un valor numérico, pero hay variación sistemática de este valor, y el parámetro adquiere un carácter dinámico. Esta variación afecta a la situación completa, la fórmula, la gráfica global, mientras que la variación de una variable 'ordinaria' solo actúa localmente.

3) El parámetro como generalizador: El parámetro se usa para generalizar sobre clases de situaciones, de casos concretos, de expresiones, fórmulas, y soluciones. El parámetro representa a estas clases y las unifica; ya no es un número específico sino que representa un número genérico o un conjunto de números, lo que permite ver lo general en lo particular, resolver categorías de problemas y formular soluciones a un nivel general. Gráficamente, al asignar valores al parámetro se obtiene un haz de gráficos.

4) El parámetro como incógnita: Ahora se trata de seleccionar casos particulares a partir de la representación general sobre la base de una condición o criterio extra. En estas situaciones el parámetro adquiere el papel de incógnita que debe ser encontrada. Esto ocurre, por ejemplo, cuando se pide resolver una ecuación, en la que intervienen parámetros, con respecto a uno de ellos; el resultado puede ser un valor, varios, o una expresión. Gráficamente, la atención de focaliza sobre un subconjunto de gráficos dentro de un haz de gráficos.

\subsection{Cuarto nivel de algebrización: uso de parámetros}

El uso de parámetros como registro numérico (placeholder) y para expresar familias de ecuaciones y funciones es indicativo de un cuarto nivel de algebrización superior al tercero nivel considerado en Godino et al. (2014), el cual está ligado a los procesos de "operar con la incógnita o con la variable". Se trata de un primer encuentro con parámetros y coeficientes variables que implica discriminación del dominio y rango de la función paramétrica, esto es, la función que asigna a cada valor del parámetro una función o ecuación específica. Como afirman Ely y Adams (2012, p. 22): "Un significativo cambio conceptual debe ocurrir para que los estudiantes se sientan seguros usando parámetros en las expresiones algebraicas en lugar de números".

\section{Ejemplo 1: La función lineal}

En la expresión algebraica, $y=2 x$, los símbolos literales $x$ e $y$ son interpretados como variables, como símbolos que pueden tomar cualquier valor del conjunto numérico previamente establecido, usualmente $R$. Los valores numéricos $x$ e $y$ pueden tomar covarían uno en términos del otro según la regla fijada en la expresión 
correspondiente, en este caso la multiplicación por 2 del valor que se asigne a $x$. El coeficiente multiplicador de $x$ se puede generalizar a un valor cualquiera dentro de un cierto dominio, lo cual se indica mediante una expresión del tipo $y=a x$; la letra $a$ interviene como un parámetro: puede tomar diferentes valores particulares pertenecientes a un cierto dominio, de manera que para cada valor que tome se obtiene una función particular, p. e., para $a=2$ tenemos $y=2 x$.

En este caso el parámetro es un símbolo literal que interviene en una expresión junto con otras variables, de tal manera que para cada valor particular que se le asigne se obtiene una función. Por tanto, es un recurso de expresión de una familia de funciones $F=\left\{f_{a}(x)=a x \mid a \in \mathbf{R}\right\}$, o de manera más precisa, de una familia de familia de funciones según el dominio $D$ de definición de las funciones $f: F_{D}=\left\{f_{a}(x)=a x \mid a \in\right.$ $\mathbf{R} ; \mathrm{x} \in D\}$.

Los símbolos literales $x$ e $y\left(f_{a}(x)\right)$ son variables indicativas de un nuevo grado de generalidad, cuyos dominios de definición y rango de variación son los conjuntos numéricos en que están definidas. El símbolo $a$ es también una variable, pero con un grado de generalidad superior, cuyo dominio de definición es el mismo u otro conjunto numérico, y el rango de valores es la familia de funciones $F_{D}$.

Así, en el ejemplo de la Tabla 2, dado que por cada alumno que va en coche $(C)$, hay tres que van a pie $(P)$, las funciones lineales que modelizan el problema son:

$$
C(x)=\frac{1}{4} x ; P(x)=\frac{3}{4} x
$$

Estas funciones tienen solución entera siempre que $x$ sea un múltiplo de 4; en particular, si $x=212$. Así, el enunciado puede ser generalizado de la siguiente forma: por cada " $a$ " alumnos que van en coche $(C)$, hay " $b$ " que van a pie $(P)(m=a+b ; a, b$ $\in \mathbf{N})$ :

$$
C_{a}(x)=\frac{a}{m} x ; P_{b}(x)=\frac{b}{m} x ; \operatorname{con} x \in\{z \in \mathbf{Z} \mid z=m k, k \in \mathbf{Z}\}
$$

Existe solución entera siempre que ax sea un múltiplo de $m$; en particular, para $x$ $=212, a=1, b=3 \mathrm{y}$, por lo tanto, $m=4$.

\section{Ejemplo 2. Ecuación cuadrática}

Los parámetros no solo se usan para expresar y operar con familias de funciones, sino también familias de ecuaciones (Ely \& Adams, 2012). Por ejemplo, $a x^{2}+b x+c=$ $0,(a \neq 0)$ es la expresión general de una familia de ecuaciones cuadráticas. Solo hay una incógnita, $x$. Las letras $a, b, c$, usualmente referidas como coeficientes que varían, toman valores específicos dentro de un conjunto de posible de valores (números reales y $c \neq 0$ ) para producir una ecuación particular.

Se dice, por tanto, que un parámetro es una variable que se usa junto con otras dos o más variables para indicar una familia de funciones o ecuaciones. En el caso de las familias de ecuaciones, el parámetro se suele denominar coeficiente. El parámetro desempeña aquí el papel de variable independiente de una función cuyo conjunto inicial está constituido por los valores del parámetro y el conjunto final por un conjunto de funciones. Por cada valor que se asigne al parámetro se obtiene como imagen una función. Por tanto, la expresión $y=a x^{2}+b x+c$, no es una función, sino una familia de funciones, aunque usualmente se menciona como "la función cuadrática". Es una 
expresión en la que intervienen tres parámetros indicados por las letras $a, b, c$. Al darle un valor particular a cada uno de los parámetros se obtiene una función cuadrática específica.

\section{Ejemplo 3. Matrices de $\boldsymbol{n}$ filas y $\boldsymbol{m}$ columnas}

Las matrices, incluso cuando son estudiadas simplemente como cajas o disposiciones ordenadas de números que resumen información estructurada, no son objetos exclusivamente aritméticos, ya que los números además del puro valor añaden un significado relativo, según el lugar que ocupan en la matriz. Además, su estudio se realiza en secundaria con cierto grado de generalidad, usando notación simbólica para representar los valores que pueden tomar los elementos incluidos en las celdas de la matriz, así como para expresar el número de filas y columnas. Como se muestra en la figura 1, la práctica discursiva mediante la que se define una matriz genérica $A=\left(a_{i j}\right)_{m, n}$ supone el uso de dos parámetros que refieren al número de filas y columnas de dicha matriz, lo que indica un nivel 4 de algebrización. El estudio de las operaciones con matrices y de sus propiedades estructurales implica un nivel superior de algebrización.

- Las matrices son tablas numéricas rectangulares:

$$
A=\left(\begin{array}{ccccc}
a_{11} & a_{12} & a_{13} & \ldots & a_{1 n} \\
a_{21} & a_{22} & a_{23} & \ldots & a_{2 n} \\
a_{31} & a_{32} & a_{33} & \ldots & a_{3 n} \\
\ldots & \ldots & \ldots & \ldots & \ldots \\
a_{m 1} & a_{m 2} & a_{m 3} & \ldots & a_{m n}
\end{array}\right) \begin{aligned}
& \text { Esta es una matriz de } m \text { filas y } \\
& \text { Los elementos, } a_{i j}, \text { son núme- } \\
& \text { ros reales }\left(a_{i j} \in \mathbb{R}\right) .
\end{aligned}
$$

Al designar una matriz genérica, como la anterior, cada término tiene dos subíndices que indican la fila y la columna a las que pertenece. El término $a_{23}$ es el que está en la segunda fila y tercera columna. Para simplificar, la matriz anterior se puede designar así:

$$
\begin{aligned}
& \left(a_{i j}\right)_{j=1, \ldots, n}^{i=1, \ldots, m} \quad \text { o bien } A=\left(a_{i j}\right)_{m, n} \text { O, simplemente, }\left(a_{i j}\right) \\
& \text { Si } m=n, \text { se dice que la matriz es cuadrada. }
\end{aligned}
$$

- Dos matrices son iguales cuando son de la misma dimensión y, además, coinciden término a término:

$$
\left.\begin{array}{l}
A=\left(a_{i j}\right)_{m, n} \\
B=\left(b_{i j}\right)_{m, n}
\end{array}\right\} \quad A=B \Leftrightarrow a_{i j}=b_{i j}
$$

- Se llama traspuesta de una matriz $A=\left(a_{i j}\right)_{m, n}$ a otra matriz $A^{t}=\left(a_{j i}\right)_{n, m}$ que se obtiene al cambiar en $A$ las filas por las columnas y las columnas por las filas.

- Una matriz $A$ se llama simétrica si $A^{t}=A$. Para que una matriz sea simétrica, necesariamente ha de ser cuadrada.

Figura 1. Matrices (Colera \& Oliveira, 2009, p. 50)

Este ejemplo sugiere la posibilidad de analizar los niveles de algebrización puestos en juego en el estudio de las matrices y su aplicación a la resolución de sistemas de ecuaciones.

\subsection{Quinto nivel de algebrización: tratamiento de parámetros}


Un nivel superior de algebrización se puede ligar a la actividad matemática desplegada cuando se realizan cálculos analíticos (sintácticos) en los que intervienen uno o más parámetros, conjuntamente con otras variables. Las operaciones con parámetros, y el establecimiento de relaciones entre ellos, conllevan una complejidad semiótica de mayor nivel dado que los objetos intervinientes y emergentes de estos sistemas de prácticas ponen en juego a los objetos algebraicos del nivel anterior (familia de ecuaciones, familia de funciones). Las operaciones que se realizan en las que intervienen parámetros, cuando son realizadas de manera comprensiva y no puramente algorítmica, implican una fase superior en el proceso de reificación de los objetos intensivos representados (familias de ecuaciones y funciones).

\section{Ejemplo 4. Obtención de la fórmula general para resolver ecuaciones de segundo grado}

Para la obtención de la fórmula general de ecuaciones cuadráticas se procede por manipulación simbólica y equivalencias sucesivas. Suponiendo que el coeficiente director $a$ es distinto de $0(a \neq 0)$ en caso contrario, la ecuación no sería de segundo grado-, se tiene:

$$
\begin{aligned}
& a x^{2}+b x+c=0 \Leftrightarrow x^{2}+\frac{b}{a} x+\frac{c}{a}=0 \Leftrightarrow x^{2}+\frac{b}{a} x=-\frac{c}{a} \Leftrightarrow \\
& \Leftrightarrow x^{2}+\frac{b}{a} x+\frac{b^{2}}{4 a^{2}}=-\frac{c}{a}+\frac{b^{2}}{4 a^{2}} \Leftrightarrow x^{2}+\frac{b}{a} x+\frac{b^{2}}{4 a^{2}}=-\frac{4 a c}{4 a^{2}}+\frac{b^{2}}{4 a^{2}} \Leftrightarrow \\
& \Leftrightarrow\left(x+\frac{b}{2 a}\right)^{2}=\frac{b^{2}-4 a c}{4 a^{2}} \Leftrightarrow x+\frac{b}{2 a}= \pm \sqrt{\frac{b^{2}-4 a c}{4 a^{2}}} \Leftrightarrow x=-\frac{b}{2 a} \pm \frac{\sqrt{b^{2} 4 a c}}{2 a} \Leftrightarrow \\
& \Leftrightarrow x=\frac{-b \pm \sqrt{b^{2}-4 a c}}{2 a}
\end{aligned}
$$

Así, en este caso la estructura de las soluciones se escriben en función de los coeficientes ligados a través de operaciones racionales (suma, resta, multiplicación, división) y cálculo de raíces cuadradas.

\section{Ejemplo 5. Progresiones geométricas}

La definición del término general de una progresión geométrica (figura 2) se realiza mediante prácticas discursivas en las cuales intervienen dos parámetros, $a_{1}$ (primer término de la sucesión) y $r$ (razón de la progresión). La sucesión es una función cuyo rango es $N$ y recorrido es $\mathbf{R}$; por tanto, los parámetros $a_{l}$ y $r$ definen una familia de funciones (sucesiones), por lo que esta práctica discursiva pone en juego un nivel 4 de algebrización. El enunciado y demostración de la propiedad que establece la suma de los $n$ primeros términos de una progresión geométrica $(r \neq 0)$ implica el cálculo con los parámetros, como se muestra en la Figura 2, por lo que implica el nivel 5 de algebrización. 


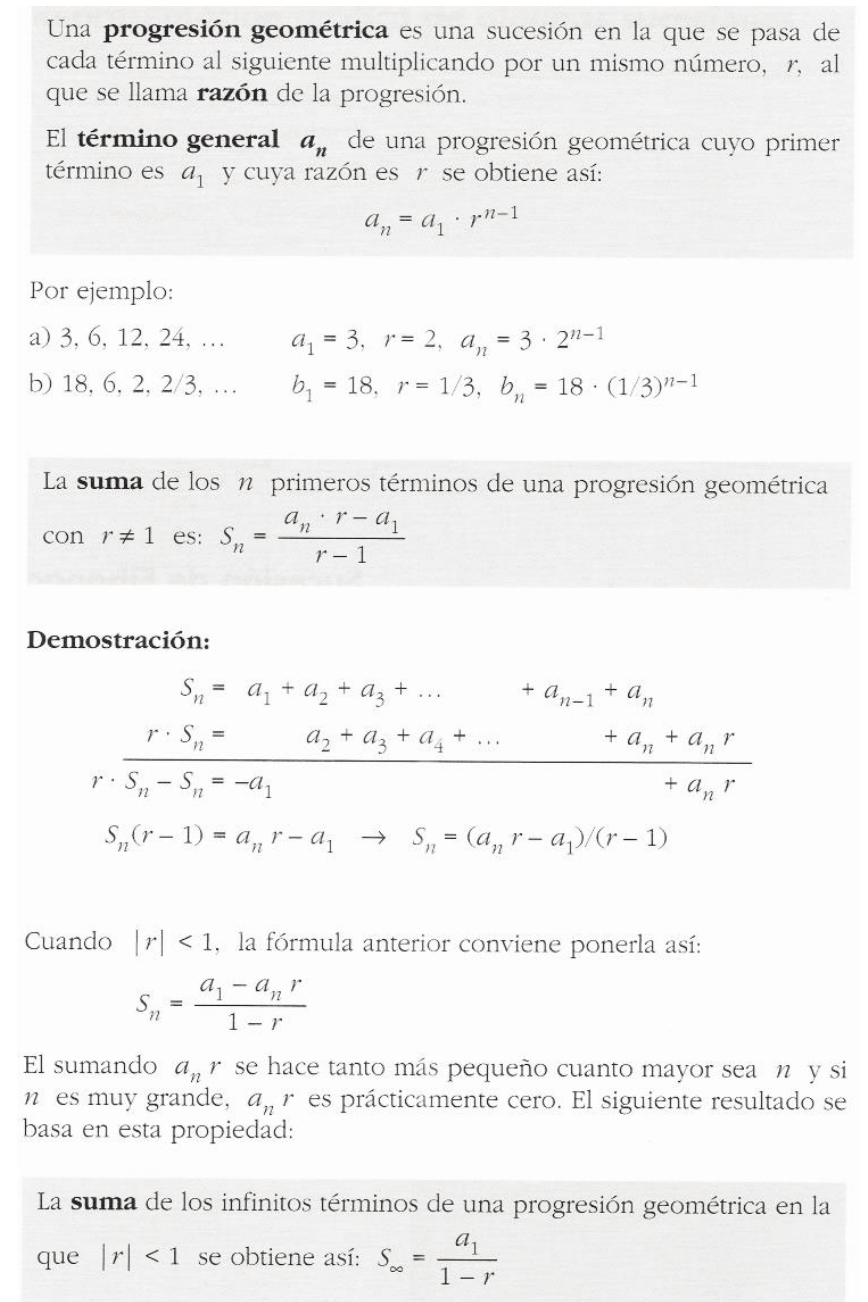

Figura 2. Progresiones geométricas (Colera, Oliveira, García \& Santaella, 2008, p. 55)

\section{Ejemplo 6. Límite finito de una función}

En la Figura 3 mostramos la definición de límite finito de una función mediante dos prácticas discursivas diferentes. En ambos casos se usa la misma notación simbólica general para designar el "paso al límite" de una función cuando la variable independiente crece indefinidamente. El crecimiento indefinido de los valores de la variable independiente $x$, dentro del dominio de definición de la función, puede tener lugar de diversas maneras, siempre que se dé a $x$ una sucesión de valores crecientes. A la generalidad con que se usa en este ejemplo el concepto de función se añade la generalidad dada al crecimiento de la variable independiente, que involucra una familia de posibles sucesiones de valores asignables a la misma. Pero en el primer caso la condición de límite para $l$ (valor fijo, pero indeterminado) se expresa con un lenguaje natural ("podemos conseguir que esté tan próximo a $l$ como queramos al dar a $x$ valores suficientemente grandes"); lenguaje que tiene un carácter fuertemente metafórico (Font, Godino, Planas, \& Acevedo, 2010). En la segunda definición se introduce de forma explícita los parámetros $\varepsilon$ y $h$, estableciendo formalmente la relación necesaria que debe existir entre ellos, lo que supone un nivel 5 de algebrización. 


$$
\begin{aligned}
\lim _{x \rightarrow+\infty} f(x)=l \Leftrightarrow & \text { Podemos conseguir que } f(x) \text { esté tan próximo a } \\
& l \text { como queramos sin más que darle a } x \text { valores } \\
& \text { suficientemente grandes. }
\end{aligned}
$$

Dicho con más precisión:

$$
\begin{aligned}
& \lim _{x \rightarrow+\infty} f(x)=l \Leftrightarrow \text { Dado un número positivo } \varepsilon \text { (arbitrariamente pe- } \\
& \text { queño), podemos encontrar un } \mathrm{h} \text { (tan grande co- } \\
& \text { mo sea necesario) tal que: } \\
& \text { si } x>\mathrm{h} \text { entonces }|f(x)-l|<\varepsilon
\end{aligned}
$$

Figura 3. Definición $\varepsilon, h$ de límite de una función (Colera \& Oliveira, 2009, p.224).

\subsection{Sexto nivel de algebrización}

La introducción de algunas estructuras algebraicas (como la de espacio vectorial, o la de grupo), el estudio del álgebra de funciones (adición, sustracción, división, multiplicación y composición) son temas que se inician en Bachillerato, poniendo en juego objetos y procesos algebraicos de mayor grado de generalidad que los considerados en el quinto nivel. Puede ser útil, por tanto, caracterizar un sexto nivel de algebrización que ayude a centrar la atención en la naturaleza específica de la actividad matemática implicada y su mayor complejidad ontosemiótica. En libros de Bachillerato encontramos textos y actividades correspondientes a este sexto nivel de algebrización:

\section{Ejemplo 7. Espacio vectorial}

En la Figura 4 encontramos una formulación general de la estructura algebraica de espacio vectorial, ejemplificada con el conjunto de matrices $M_{\mathrm{m}, \mathrm{n}}$, y el conjunto de las $n$ tuplas de números reales, $R^{\mathrm{n}}$.

\section{Espacios vectoriales}

La idea de vector como flecha da lugar a la de espacio vectorial: conjunto de todos los vectores entre los cuales se definen unas operaciones que cumplen ciertas propiedades. Pero hay otros entes matemáticos con las mismas operaciones y propiedades. Por eso, la definición de espacio vectorial es mucho más amplia y abierta que una colección de "flechas".

\begin{tabular}{|c|c|}
\hline \multicolumn{2}{|r|}{ SUMA DE VECTORES } \\
\hline ASOCIATIVA & $(\vec{u}+\vec{v})+\vec{w}=\vec{u}+(\vec{v}+\vec{w})$ \\
\hline CONMUTATIVA & $\overrightarrow{\mathrm{u}}+\overrightarrow{\mathrm{v}}=\overrightarrow{\mathrm{v}}+\overrightarrow{\mathrm{u}}$ \\
\hline VECTOR NULO & $\begin{array}{l}\text { Es un vector llamado } \overrightarrow{0} \text { tal que si } \vec{v} \in V \text { cumple: } \\
\qquad \vec{v}+\overrightarrow{0}=\vec{v}\end{array}$ \\
\hline VECTOR OPUESTO & Todo $\vec{v}$ tiene un opuesto, $-\vec{v}: \vec{v}+(-\vec{v})=\overrightarrow{0}$ \\
\hline \multicolumn{2}{|r|}{ PRODUCTO DE UN NÚMERO POR UN VECTOR } \\
\hline ASOCIATIVA & $(a \cdot b) \cdot \overrightarrow{\mathrm{v}}=a \cdot(b \cdot \overrightarrow{\mathrm{v}})$ \\
\hline DISTRIBUTIVA I & $(a+b) \cdot \vec{v}=a \cdot \vec{v}+b \cdot \vec{v}$ \\
\hline DISTRIBUTIVA II & $a \cdot(\overrightarrow{\mathrm{u}}+\overrightarrow{\mathrm{v}})=a \cdot \overrightarrow{\mathrm{u}}+a \cdot \overrightarrow{\mathrm{v}}$ \\
\hline PRODUCTO POR 1 & Si $\vec{v} \in V$ se cumple que $1 \cdot \vec{v}=\vec{v}$ \\
\hline
\end{tabular}

Tenemos un conjunto, $V$, entre cuyos elementos (a los que llamaremos vectores) hay definidas dos operaciones:

SUMa DE DOS ELEMENTOS DE $V: \quad$ si $\overrightarrow{\mathrm{u}}, \overrightarrow{\mathrm{v}} \in V$, entonces $\overrightarrow{\mathrm{u}}+\overrightarrow{\mathrm{v}} \in V$

PRODUCTO POR UN NÚMERO REAL: si $a \in \mathbb{R}$ y $\overrightarrow{\mathrm{u}} \in V$, entonces $a \cdot \overrightarrow{\mathrm{u}} \in V$

Se dice que $(V,+, \cdot)$ es un espacio vectorial sobre $\mathbb{R}$ si las operaciones cumplen las siguientes propiedades:

Figura 4. Espacio vectorial (Colera \& Oliveira, 2009, p. 62) 
Se trata de un primer encuentro con la estructura algebraica de espacio vectorial en la que se pone en juego un conjunto de objetos matemáticos (vectores) sobre los cuales se definen operaciones que cumplen un sistema de propiedades específicas. Esto exige un primer "estudio estructural" del conjunto de los vectores, ya que en este tipo de presentación (axiomática) se explicitan los invariantes de las propiedades de la suma de vectores (existencia de un mismo vector para todos que no produce cambios, existencia de un vector opuesto para cualquier vector, etc.). Además, se estudian relaciones entre las propiedades de los elementos que caracterizan un vector y los números reales (escalares), funcionando éstos como parámetros.

\section{Ejemplo 8: Composición de funciones}

En el caso de la Figura 5 se pone en juego la noción de función en toda su generalidad, no concretada en una familia de funciones particulares sino refiriéndose a una función cualquiera y se opera con ella para producir otra nueva función y estudiar sus propiedades; en particular, se determina que la composición de funciones no es conmutativa.

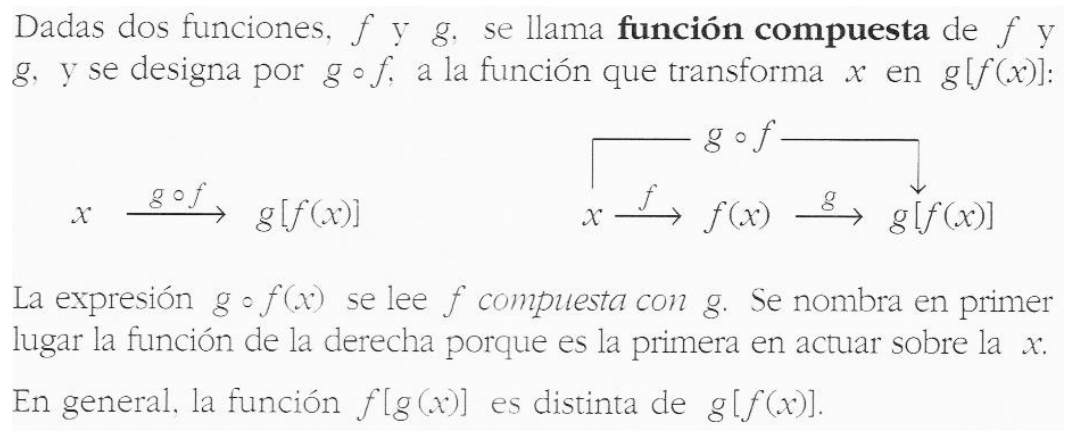

Figura 5. Composición de funciones (Colera et al., 2008, p. 256)

De hecho, un conjunto de funciones (polinómicas, por ejemplo) dotado de ciertas operaciones (adición, multiplicación, etc.) constituye un "álgebra".

\subsection{Niveles de algebrización, objetos y procesos matemáticos}

Los niveles de algebrización son básicamente grados de generalidad, combinada con el uso de diversos registros de representación semiótica (RRS), sus transformaciones y conversiones (Duval, 1995), los cuales son indicativos de fases en el proceso de reificación de los objetos intensivos intervinientes. Consideramos que para la descripción del carácter algebraico de las prácticas matemáticas es útil fijar la atención en los objetos resultantes de los procesos de generalización, y del proceso dual de particularización. Como resultado de los procesos de generalización obtenemos objetos matemáticos que se denominan objetos intensivos en el marco del EOS, que vienen a ser la regla que genera la clase, el tipo o generalidad implicada (Godino, Font, Wilhelmi y Lurduy, 2011). En el ejemplo 1, el caso particular (1 de cada 4 van en coche) se generaliza para describir una familia de funciones $\left(C_{a}(x)=\frac{a}{m} x\right)$.

Mediante los procesos inversos de particularización se obtienen objetos que se denominan extensivos, esto es, objetos particulares. Así sucede en el ejemplo 5, donde 
se ilustra la noción de progresión geométrica con valores concretos $\left(a_{1}=3, r=2\right)$. El objeto intensivo puede ser visto como la regla que genera los elementos que componen una colección o conjunto, sea finito o infinito. Una colección finita simplemente enumerada no se debe considerar como un intensivo hasta el momento en que el sujeto muestra el criterio o regla que se aplica para delimitar los elementos constituyentes del conjunto. Entonces el conjunto pasa a ser algo nuevo, diferente de los elementos que lo constituyen, como una entidad unitaria emergente del sistema. Por tanto, además de la generalización que da lugar al conjunto, hay un proceso de unitarización. En el mismo ejemplo 5, tras la particularización, se establece la suma de los $n$ primeros términos, que supone la determinación de una propiedad de un nuevo objeto único: "la" progresión geométrica.

Por otra parte, la nueva entidad unitaria tiene que ser hecha ostensiva o materializada mediante un nombre, icono, gesto o un símbolo, a fin de que pueda participar de otras prácticas, procesos y operaciones. El objeto ostensivo que materializa al objeto unitario emergente de la generalización es otro objeto que refiere a la nueva entidad intensiva, por lo que tiene lugar un proceso de representación que acompaña la generalización y materialización. Finalmente, el símbolo se desprende de los referentes a los cuales representa/sustituye para convertirse en objeto sobre el cual se realizan acciones (proceso de reificación). Estos símbolos-objetos forman nuevos conjuntos sobre los cuales se definen operaciones, propiedades y estructuras, esto es, sobre los cuales se opera de manera sintáctica, analítica o formal (Godino, et al., 2014). En el ejemplo 5, la demostración de la fórmula de la suma de los $n$ primeros términos implica la manipulación sintáctica de los símbolos, lo cual supone una reificación de la progresión en tanto que objetos lingüísticos.

La manipulación o cálculo con parámetros, de manera comprensiva por parte del sujeto, implica la intervención de los procesos de unitarización, materialización, reificación y representación sobre objetos intensivos de mayor generalidad que los llevados a cabo cuando intervienen variables no paramétricas. En el ejemplo 6 descrito anteriormente sobre el límite finito de una función se muestra con claridad el contraste entre las dos definiciones dadas, una usando un lenguaje natural metafórico y otra con lenguaje simbólico $(\varepsilon, h)$, que muestra la intervención de "parámetros" y su uso operativo para dar precisión a la definición de límite.

El uso de dispositivos de cálculo simbólico y representación gráfica como, por ejemplo, GeoGebra (GGB), permite manipular con facilidad expresiones algebraicas en las que intervienen variables y parámetros, $\mathrm{y}$, por tanto, familias de ecuaciones y funciones. Las conversiones entre los registros de representación semiótica tabular, gráfica y simbólica de los objetos algebraicos pueden suponer una ayuda en las primeras fases del proceso de reificación de tales objetos. Por ejemplo, en el applet de la figura 6 se articulan los marcos numérico, simbólico y gráfico con GGB, ya que la manipulación mediante "deslizadores" de los coeficientes de las funciones afines $f$ y $g$ tiene efecto en los tres marcos.

A pesar de la gran versatilidad de GGB, el reconocimiento, comprensión y dominio competente de los objetos algebraicos como entidades unitarias, propios de la fase estructural del proceso de reificación, puede requerir la implementación de momentos regulativos o de institucionalización, que articulen y complementen los momentos de ilustración, exploración y demostración con GGB (Lasa y Wilhelmi, 2013). Asimismo, tampoco se deben subestimar las dificultades potenciales que pueden tener los 
estudiantes para convertir los artefactos manipulativos en verdaderos instrumentos de trabajo matemático.

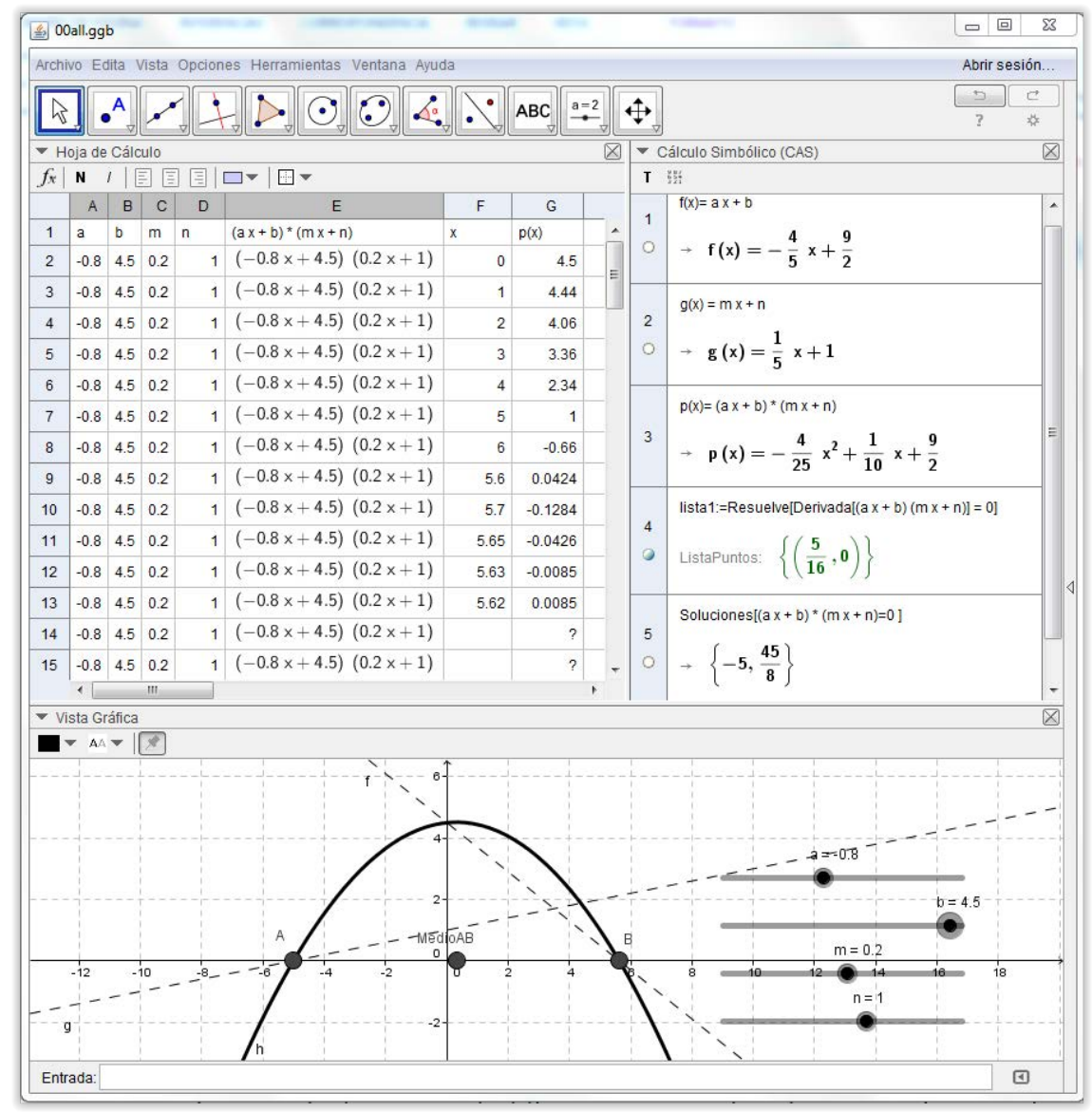

Figura 6. Articulación de marcos numérico, simbólico y gráfico con GGB (http://tube.geogebra.org/material/show/id/1144601)

\section{Etapas del proceso de algebrización en el marco de la teoría antropológica}

En el marco de la Teoría Antropológica de lo Didáctico (TAD) (Chevallard, 1992; 1999) se ha propuesto un modelo epistemológico del álgebra en Educación Secundaria que distingue tres etapas (o niveles) en el proceso de algebrización de la actividad matemática.

\subsection{Primera etapa del proceso de algebrización}

Ruiz-Munzón, Bosch y Gascón (2010) consideran como "problema aritmético" aquellos problemas que pueden resolverse mediante una cadena de operaciones aritméticas $(+,-, \times, /$, etc.) ejecutables a partir de los datos del problema, datos que acostumbran a ser cantidades conocidas de ciertas magnitudes. Como ejemplo de problema que se resuelve mediante un "programa de cálculo aritmético" (PCA) indican el siguiente enunciado:

P0: Gabriel piensa un número, le suma 25, divide el resultado entre 2, resta 8 y lo multiplica todo por 3. Si al final obtiene 21, ¿qué número pensó Gabriel? 
Una resolución aritmética, expresada de manera verbal, sería: "Si al final obtiene 21 , antes de multiplicar por 3 tenía 7, antes de restarle 8 tenía 15, antes de dividir entre 2 tenía 30 y antes de sumar 25 tenía 5. Luego Gabriel pensó el número 5".

En el modelo EOS esta actividad matemática sería de nivel 0 de algebrización. No obstante, si el resolutor plantea y resuelve la ecuación que traduce la secuencia de pasos del PCA de manera simbólica - literal la actividad matemática realizada sería de nivel 2.

$$
\left(\frac{n+25}{2}-8\right) 3=21 ; 3 n+75-48=42 ; 3 n=15 ; n=5
$$

Los problemas del siguiente tipo son propios de una primera etapa de algebrización:

Pla: Piensa un número, súmale el doble de su consecutivo, suma 15 al resultado y, por último, resta el triple del número pensado inicialmente. ¿Qué resultado has obtenido? Repite el proceso con otro número diferente ¿Se obtiene siempre el mismo número? ¿Por qué?

Según Ruiz-Munzón et al. (2010) la resolución de este tipo de problemas lleva a identificar una primera etapa del proceso de algebrización con el momento en que es necesario considerar el PCA como un todo, es decir, producir una formulación escrita (simbólica) del PCA, que ya es, en cierto sentido, una expresión algebraica. Aparece entonces la necesidad de construir nuevas técnicas, esencialmente de "simplificación", para trabajar sobre las expresiones algebraicas. Se define una expresión algebraica como la formulación simbólica de un PCA que, en general, se puede utilizar para modelizar tanto el proceso de resolución de un problema aritmético como su estructura. Por "simplificar un PCA" se entiende la operación de transformarlo en otro equivalente y que, en cierto sentido, sea más "sencillo" o más "adaptado" para utilizarlo en una actividad matemática concreta.

Para resolver el problema hay que operar con la variable (simplificar una expresión simbólica), pero no trasponer términos porque la variable está en un solo término de la igualdad: $n+2(n+1)+15-3 n=f(n)=17$

Aquí $n$ no es un número desconocido (incógnita) que haya que determinar según las condiciones del problema, sino una variable que puede tomar diversos valores. Para cada valor que se dé a $n$, se tiene una ecuación $f(n)=17$. Para el modelo EOS esta actividad también sería de nivel 2 de algebrización. Es claro que al preguntar si se obtiene siempre el mismo resultado y pedir una justificación se está requiriendo una generalización, lo que lleva a que la tarea en sí misma no pueda ser considerada como aritmética. Sin embargo, también se puede resolver de manera aritmética pensando de la siguiente manera, en cuyo caso la actividad seguiría siendo de nivel 0 :

El doble del número consecutivo del número es el doble del número más 2; 2 más 15 es 17; el número más su doble es el triple del número. Como en la secuencia de operaciones también se pide restar el triple del número el valor final que se obtiene es 17 , cualquiera que sea el número que haya pensado.

Ruiz-Munzón et al. (2010) incluyen en la primera etapa de algebrización la siguiente variante del problema P1a:

P1b: Noelia y Marga piensan, independientemente, sendos números. Noelia multiplica su número por 3, resta 18 y acaba dividiendo este resultado entre 9. Marga resta 4 al número que pensó, a continuación multiplica el resultado por 5 y acaba dividiendo el resultado por 10. Si, casualmente, obtienen el mismo resultado final, ¿qué relación hay entre los números pensados por Noelia y Marga? 
La traducción del enunciado verbal a simbólico - literal sería,

$$
\frac{3 n-18}{9}=\frac{(m-4) 5}{10}
$$

Simplificando se obtiene, $n=(3 / 2) m$, que es una relación funcional entre dos variables. Para nosotros sería una actividad de nivel 2; se opera con las variables sólo con técnicas de simplificación, no de cancelación.

Como conclusión podemos afirmar que la primera etapa del proceso de algebrización en el modelo de la TAD implica una actividad de nivel 2 en el modelo basado en EOS.

\subsection{Segunda etapa del proceso de algebrización}

El paso a la segunda etapa del proceso de algebrización se identifica con la necesidad de igualar dos PCA. Se requiere de nuevas técnicas, las técnicas de cancelación, puesto que hay que manipular una igualdad de dos PCA como un nuevo objeto matemático (ecuación). Dichas técnicas tienen por objeto obtener "ecuaciones equivalentes" y no sólo PCA equivalentes como pasaba con las técnicas de simplificación características de M1. Aparece así un segundo modelo M2 que, además de aumentar el nivel de algebrización, amplia y completa M1. Veamos un problema que se sitúa plenamente en $\mathrm{M} 2$ :

P2: Judit piensa dos números positivos $n$ y $m(m>n)$. Al triple de $n$ le resta la diferencia entre $m$ y $n$. Ahora a $m$ le suma $n$, le suma el triple de $m$ y finalmente suma 2 al resultado. Si el resultado de las dos secuencias de operaciones coincide, ¿qué relación existe entre $n$ y $m$ ?

La traducción del enunciado verbal a simbólico - literal sería:

$$
3 n-(m-n)=m+n+3 m+2 ;(m-n)=2 n-4 m-2 ; 5 m=3 n-2 ; m=(3 n-2) / 5
$$

En este problema se está pidiendo encontrar una relación (función afín) entre las variables $n$ y $m$; para obtener la expresión simplificada del criterio de dicha función es necesario operar con las variables, aplicando técnicas de cancelación, ya que ambas variables están incluidas en los dos términos de la igualdad. Se trata de una actividad matemática con un nivel 3 de algebrización según el modelo EOS, mientras que en el modelo TAD corresponde a la etapa 2 .

\subsection{Tercera etapa del proceso de algebrización según la TAD}

En el modelo de la TAD se considera como tercera etapa del proceso de algebrización el momento en que se requiere una fuerte generalización de los problemas de M2 debido a la necesidad de no limitar el número de variables y de no distinguir entre incógnitas y parámetros. Por ejemplo, el siguiente problema ya no forma parte de M2:

P3: ¿Qué relación hay entre el perímetro P y el área A de un triángulo isósceles? ¿En qué casos $P$ y A determinan un único triángulo isósceles?

Llamando $b$ a la longitud del lado desigual y $a$ la de los dos lados iguales, $\mathrm{P}$ al perímetro y A al área del triángulo se tiene,

$$
P=2 a+b ; a=\frac{(P-b)}{2}
$$




$$
A=\frac{1}{2} b \sqrt{a^{2}-\frac{b^{2}}{4}}
$$

Sustituyendo y simplificando se obtiene la relación, dependiendo del parámetro $b$ :

$$
16 A^{2}=P^{2} b^{2}-2 P A b^{3}
$$

El problema P3 que se presenta como indicativo de la tercera etapa de algebrización implica el uso y tratamiento de los parámetros $a$ y $b$ (longitudes de los lados del triángulo), lo que sin duda supone un alto nivel de complejidad ontosemiótica.

En la matemática de secundaria hay una cierta variedad de situaciones y tareas que implican la introducción de parámetros para indicar familias de funciones, pero que no precisan el tratamiento de las expresiones correspondientes. Parece conveniente, en consecuencia, con el propósito de describir la actividad matemática en Educación Secundaria, definir un nivel intermedio de algebrización entre la etapa 2 y 3 del modelo de la TAD, los cuales en el modelo EOS corresponderían a niveles 4 y 5 .

Por último, consideramos que comprender el funcionamiento de una estructura, operar con funciones u obtener nuevas funciones a partir de analizar propiedades de las mismas exige considerar un nuevo nivel de algebrización al que identificamos como nivel 6. Actividades de este tipo aparecen en textos de Bachillerato como se ha indicado (Sección 3.3).

\section{Síntesis e implicaciones para la formación de profesores}

En este artículo hemos completado el trabajo iniciado en Aké et al. (2013) y Godino et al. (2014) sobre la identificación de niveles de algebrización de la actividad matemática en Educación Primaria, incluyendo tres nuevos niveles que caracterizan la matemática de Secundaria (incluido Bachillerato). Como síntesis proponemos los siguientes seis niveles de razonamiento algebraico en primaria y secundaria (junto con el nivel 0 indicativo de ausencia de algebrización):

- Nivel 0. Se opera con objetos intensivos de primer grado de generalidad, usando lenguajes natural, numérico, icónico, gestual.

- Nivel 1. Se usan objetos intensivos de segundo grado de generalidad, propiedades de la estructura algebraica de $\mathrm{N}$ y la igualdad como equivalencia.

- Nivel 2. Se usan representaciones simbólico - literales para referir a los objetos intensivos reconocidos, los cuales están ligados a la información espacial, temporal y contextual; se resuelven ecuaciones de la forma $A x+B=C(A, B, C$ $\in \mathbf{R})$.

- Nivel 3. Los símbolos se usan de manera analítica, sin referir a la información contextual. Se realizan operaciones con indeterminadas o variables; se resuelven ecuaciones de la forma $A x+B=C x+D(A, B, C, D \in \mathbf{R})$.

- Nivel 4. Se estudian familias de ecuaciones y funciones usando parámetros y coeficientes.

- Nivel 5. Se realizan cálculos analíticos (sintácticos) que implican el uso de uno más parámetros, junto con variables o indeterminadas. 
- Nivel 6. Se comienza a estudiar estructuras algebraicas en sí mismas, sus definiciones y propiedades estructurales.

Es posible que entre los niveles 4 y 5 de algebrización se pueda identificar un nivel intermedio que refleje una fase intermedia de reificación de los objetos intensivos correspondientes. De igual modo, es posible que el nivel 6 de algebrización, cuya descripción refleja una fase incipiente de reificación de los objetos intensivos intervinientes, se pueda complementar con otros dos niveles más avanzados, propios de los estudios universitarios. Esta es una cuestión abierta a futuras investigaciones.

Por otro lado, se ha iniciado el estudio de la articulación de este modelo con las etapas del proceso de algebrización definidas en el marco de la Teoría Antropológica de lo Didáctico (Bolea, 2002; Gascón, 1999, 2011; Ruiz-Munzón, Bosch \& Gascón, 2010) con los niveles de algebrización en el EOS. A pesar de que las "etapas" según la TAD se refieren a recorridos de estudio potenciales y los "niveles" según el EOS a la actividad efectivamente desarrollada por los sujetos, es posible establecer paralelismos entre ambos modelos. Así, se concluye que el nivel 2 del modelo basado en EOS se corresponde con la etapa 1 de la TAD y la etapa 2 de la TAD se corresponde con el nivel 3 del EOS. La etapa 3 del proceso de algebrización propuesta por la TAD, analizada desde la perspectiva del EOS, lleva a proponer la distinción de dos niveles adicionales ( $4^{\circ}$ y $5^{\circ}$ en el modelo EOS), al tener en cuenta la complejidad ontosemiótica del uso de parámetros para expresar familias de ecuaciones y funciones. Por último, el nivel 6 del EOS propuesto no se corresponde de manera explícita con un nivel en el modelo basado en la TAD. En la Tabla 3 se resumen estas relaciones.

Tabla 3. Correspondencia entre las etapas (TAD) y los niveles (EOS) de algebrización

\begin{tabular}{ll}
\hline \multicolumn{1}{c}{ EOS } & \multicolumn{1}{c}{ TAD } \\
\hline Nivel 0: aritmético & PCA: programa de cálculo aritmético \\
Nivel 1: proto-algebraico incipiente & Etapa 1: necesidad de formulación simbólica de un PCA \\
Nivel 2: proto-algebraico intermedio & Etapa 2: igualación de dos PCA \\
Nivel 3: algebraico consolidado & Etapa 3: introducción de parámetros \\
Nivel 4: uso de parámetros & \\
Nivel 5: manipulación de parámetros & \\
Nivel 6: tareas estructurales & \\
\hline
\end{tabular}

Los tipos de objetos y procesos algebraicos considerados en el modelo descrito en este trabajo suponen un análisis microscópico complementario al abordado mediante la noción de praxeología usado en el marco de la TAD. De hecho, el modelo propuesto permite un estudio pormenorizado de los comportamientos de los sujetos, que amplía el carácter institucional abordado desde la identificación de las praxeologías matemáticas y didácticas. Además, al incluir nuestro modelo niveles proto-algebraicos, está más adaptado a etapas donde el razonamiento algebraico es incipiente (tercer ciclo de Educación Primaria y primer ciclo de Educación Secundaria), mientras que las sucesivas etapas del proceso de algebrización propuestas desde la TAD en los trabajos citados, están más centradas en la caracterización del álgebra en niveles educativos superiores. Así, aunque los criterios usados en ambos modelos teóricos para definir niveles o etapas en el proceso de algebrización son distintos, el análisis teórico realizado muestra que son consistentes y complementarios, reconociendo, no obstante, que la plena articulación de estos modelos deberá ser profundizada en estudios posteriores. 
El estudio realizado sobre la naturaleza del razonamiento algebraico tiene implicaciones para la formación de profesores, tanto en Educación Primaria como en Educación Secundaria. No basta con elaborar propuestas curriculares (NCTM, 2000) que incluya el álgebra desde los primeros niveles educativos, se precisa que el docente actúe como principal agente de cambio en la introducción y desarrollo del razonamiento algebraico en las aulas de primaria, y de su progresión en secundaria. El análisis focalizado en el reconocimiento de objetos y procesos propios del pensamiento algebraico, puede facilitar la identificación de rasgos de las prácticas matemáticas sobre los cuales se puede intervenir para aumentar progresivamente el nivel de algebrización de la actividad matemática de los alumnos. El comienzo de un trabajo de este tipo en la formación inicial de maestros de educación primaria se describe en Aké, Godino, Fernández y Gonzato (2014).

El reconocimiento de los niveles 4, 5 y 6 de algebrización por parte de los profesores de Educación Secundaria, junto con la articulación de los niveles previos, pretende ayudar a tomar conciencia de las brechas o discontinuidades ontosemióticas que pueden tener lugar en la realización de las tareas que se proponen a los estudiantes.

Este artículo contribuye a la caracterización de los niveles de algebrización de la actividad matemática escolar. Los niveles podrían ser usados como potenciales explicaciones de algunos conflictos en el aprendizaje del álgebra escolar y ofrecer criterios para el diseño curricular e instruccional. En estos momentos solo podemos formular como hipótesis que algunas dificultades podrían ser explicadas porque el diseño curricular, las lecciones de los libros de texto y la actuación de los profesores en el aula no tienen en cuenta, con la atención necesaria, la complejidad de objetos y procesos que se ponen en juego en las prácticas matemáticas escolares.

Queda pues abierta la elaboración de propuestas de enseñanza y aprendizaje que contemplen el progreso de los estudiantes en los distintos niveles. Este progreso no es lineal. Lasa y Wilhelmi (2015) muestran la importancia de la adaptación de los procedimientos a la tarea. En particular, aportan datos experimentales de estudiantes de $2^{\circ}$ ESO con una competencia algebraica de nivel 3 que realizan tareas combinatorias de recuento mediante procedimientos exclusivamente aritméticos mejor adaptados a la tarea, renunciando pues a su maestría algebraica y mostrando, por lo tanto, un uso flexible de los procedimientos aritméticos y algebraicos. Así, las propuestas tendrán que venir acompañadas de instrumentos de evaluación de su idoneidad didáctica (Godino, Bencomo, Font y Wilhelmi, 2006).

El reconocimiento de los niveles de algebrización de la actividad matemática puede ayudar a tomar conciencia de brechas o discontinuidades en la secuencia de configuraciones que componen las trayectorias epistémicas de los correspondientes procesos de estudio matemático (Godino, Contreras \& Font, 2006). Tales brechas se refieren al uso de distintos registros de representación semiótica, su tratamiento y conversión, así como a la intervención y puesta en relación de objetos conceptuales, proposicionales, procedimentales y argumentativos de mayor grado de generalidad. Asimismo, la identificación de los objetos, procesos y significados que suponen el acceso a los distintos niveles de algebrización puede permitir el diseño de prácticas operativas, discursivas y regulativas cuyo objetivo es la progresión del aprendizaje. El contraste de estos supuestos es una cuestión abierta a futuras investigaciones.

\section{Agradecimientos.}


Trabajo realizado en el marco de los proyectos de investigación, EDU2012-31869 y EDU2013-41141-P, Ministerio de Economía y Competitividad (MINECO).

\section{Referencias}

Aké, L. Godino, J. D., Gonzato, M., \& Wilhelmi, M. R. (2013). Proto-algebraic levels of mathematical thinking. En A. M. Lindmeier \& A. Heinze (Eds.), Proceedings of the 37th Conference of the International Group for the Psychology of Mathematics Education (Vol. 2, pp. 1-8). Kiel, Germany: IGPME.

Aké, L., Godino, J. D., Fernández, T., \& Gonzato, M. (2014), Ingeniería didáctica para desarrollar el sentido algebraico de maestros en formación. Avances de Investigación en Educación Matemática, 5, 25 - 48.

Bolea, P. (2002). El proceso de algebrización de organizaciones matemáticas escolares. Tesis doctoral. Universidad de Zaragoza.

Cai, J., \& Knuth, E. (2011). Early algebraization. A global dialogue from multiple perspectives. Berlin: Springer-Verlag.

Carraher, D. W., \& Schliemann, A. L. (2007). Early algebra and algebraic reasoning. En: F. Lester (Ed.), Second handbook of research on mathematics teaching and learning (Vol. 2, 669-705). Charlotte, N.C: Information Age. y NCTM.

Caspi, S., \& Sfard, A. (2012). Spontaneous meta-arithmetic as a first step toward school algebra. International Journal of Educational Research, 51-52, 45-65.

Chevallard, Y. (1992), Concepts fondamentaux de la didactique: Perspectives apportées par une approche anthropologique. Recherches en Didactique des Mathématiques, $12(1), 73-112$.

Chevallard, Y. (1999). L'analyse des pratiques enseignantes en théorie anthropologique du didactique. Recherches en Didactique des Mathématiques, 19 (2), 221-266.

Chevallard, Y., \& Bosch, M. (2012). L'algèbre entre effacement et réaffirmation. Aspects critiques de l'offre scolaire d'algèbre. En L. Coulange, J.-P. Drouhard, J. L. Dorier, \& A. Robert (Coord.), Enseignement de l'algèbre élémentaire. Bilan et perspectives. Recherches en Didactique des Mathématiques, special issue, 13-33.

Colera, J., \& Oliveira, M. J. (2009). Matemáticas II. Bachillerato. Madrid: Anaya.

Colera, J., Oliveira, M. J., García, R., \& Santaella, E. (2008). Matemáticas I. Bachillerato. Madrid: Anaya.

Drijvers, P. (2003). Learning algebra in a computer algebra environment: design research on the understanding of the concept of parameter. Universidad de Utrecht. Disponible en, http://dspace.library.uu.nl/handle/1874/886

Duval, R. (1995). Sémiosis et penseé humaine. Berna: Peter Lang.

Ely, R., \& Adams, A. (2012). Unknown, placeholder, or variable: what is $\mathrm{x}$ ? Mathematics Education Research Journal, 24 (1), 199-38.

Filloy, E., \& Rojano, T. (1989). Solving equations: The transition from arithmetic to algebra. For the Learning of Mathematics, 9 (2), 19-25.

Filloy, E., Puig, L. \& Rojano, T. (2008). Educational algebra. A theoretical and empirical approach. New York: Springer. 
Font, V., Godino, J. D., Planas, N.. \& Acevedo, J. I. (2010). The object metaphor and sinecdoque in mathematics classroom discourse. For the Learning of Mathematics, $30,15-19$.

Gascón, J. (1999). La naturaleza prealgebraica de la matemática escolar. Educación Matemática, 11(1), 77-88.

Gascón, J. (2011). Las tres dimensiones fundamentales de un problema didáctico. El caso del álgebra elemental. Revista Latinoamericana de Investigación en Matemática Educativa, 14 (2), 203-231.

Godino, J. D. (2012). Origen y aportaciones de la perspectiva ontosemiótica de investigación en Didáctica de la Matemática. En A. Estepa, A. Contreras, J. Deulofeu, M. C. Penalva, F. J. García. \& L. Ordóñez (Eds.), Investigación en Educación Matemática XVI (pp. 49 - 68). Jaén: SEIEM.

Godino, J. D., Batanero, C., \& Font, V. (2007). The onto-semiotic approach to research in mathematics education. ZDM. The International Journal on Mathematics Education, 39 (1-2), 127-135.

Godino, J. D., Contreras, A., \& Font, V. (2006). Análisis de procesos de instrucción basado en el enfoque ontológico-semiótico de la cognición matemática. Recherches en Didactiques des Mathématiques, 26 (1), 39-88.

Godino, J. D., Bencomo, D., Font, V., \& Wilhelmi, M. R. (2006). Análisis y valoración de la idoneidad didáctica de procesos de estudio de las matemáticas. Paradigma, XXVII (2), 221-252.

Godino, J. D., Font, V., Wilhelmi, M. R., \& Lurduy, O. (2011). Why is the learning of elementary arithmetic concepts difficult? Semiotic tools for understanding the nature of mathematical objects. Educational Studies Mathematics, 77 (2), 247-265.

Godino, J. D. Aké, L., Gonzato, M., \& Wilhelmi, M. R. (2014). Niveles de algebrización de la actividad matemática escolar. Implicaciones para la formación de maestros. Enseñanza de las Ciencias, 32 (1), 199-219.

Lasa, A., \& Wilhelmi, M. R. (2013). Use of GeoGebra in explorative, illustrative and demonstrative moments. Revista do Instituto GeoGebra de São Paulo, 2 (1), 52-64. Recuparable en

http://revistas.pucsp.br/index.php/IGISP/article/viewFile/15160/12279.]

Lasa, A., \& Wilhelmi, M. R. (2015). Atando cabos, contando circunferencias. En J. M. Contreras, C. Batanero, J. D. Godino, G.R. Cañadas, P. Arteaga, E. Molina, M.M. Gea y M.M. López (Eds.), Didáctica de la Estadística, Probabilidad y Combinatoria 2. (pp. 145-152). Granada: SEIEM.

Kaput, J. (2008). What is algebra? What is algebraic reasoning? En J. Kaput, D. W. Carraher, y M. L. Blanton (Eds), Algebra in the early grades (pp. 5-17). New York: Routledge.

Kieran, K. (2007). Learning and teaching algebra at the middle school through college levels. Building meaning for symbols and their manipulation. En, F. Lester (Ed.), Second handbook of research on mathematics teaching and learning (Vol. 2, 707762). Charlotte, N.C: Information Age y NCTM.

National Council of Teachers of Mathematics (NCTM) (2000). Principles and standards for school mathematics. Reston, VA: Author. 
Radford, L. (2011). Grade 2 students' non-symbolic algebraic thinking. En J. Cai, \& E. Knuth (Eds.), Early algebraization. Advances in mathematics education (pp. 303322). Berlin: SpringerVerlag.

Ruiz-Munzón, N., Bosch, M., \& Gascón, J. (2010). La algebrización de los programas de cálculo aritmético y la introducción del álgebra en secundaria. En M. M. Moreno, A. Estrada, J. Carrillo, \& T.A. Sierra, (Eds.), Investigación en Educación Matemática XIV (pp. 545-556). Lleida: SEIEM.

Ruiz-Munzón, N. Bosch, M., \& Gascón (2011). Un modelo epistemológico de referencia del álgebra como instrumento de modelización. En Bosch, M., Gascón, J., Ruiz Olarría, A., Artaud, M., Bronner, A., Chevallard, Y., Cirade, G., Ladage, C., \& Larguier, M. (Eds.), Un panorama de la TAD. (pp. 743-765). III Congreso Internacional sobre la TAD.

Sfard, A. \& Linchevskin, L. (1994). The gains and the pitfalls of reificación - The case of algebra. Educational Studies in Mathematics 26, 191-228.

Socas, M. (2011). La enseñanza del álgebra en la educación obligatoria. Aportaciones de la investigación. Números 77, 5-34.

\section{Referencias a los autores}

Juan D. Godino, Universidad de Granada (España), jgodino@ugr.es

Teresa Neto, Centro de Investigación "Didáctica y Tecnología en la Formación de Profesores", CIDTFF, Universidad de Aveiro (Portugal), teresaneto@ua.pt

Miguel R. Wilhelmi, Universidad Pública de Navarra (España), miguelr.wilhelmi@unavarra.es

Lilia Aké, Universidad de Colima (México), liliapatricia_ake@ucol.mx

Silvia Etchegaray, Universidad Nacional de Río Cuarto (Argentina), setchegaray@exa.unrc.edu.ar

Aitzol Lasa, Universidad Pública de Navarra (España), aitzol.lasa@unavarra.es 


\title{
Algebrization levels of school mathematics practices. Networking of the Onto-semiotic and Anthropological perspectives
}

\author{
Juan D. Godino, Universidad de Granada (España) \\ Teresa Neto, Centro de Investigación "Didáctica y Tecnología en la Formación de \\ Profesores", CIDTFF, Universidad de Aveiro (Portugal) \\ Miguel R. Wilhelmi, Universidad Pública de Navarra (España) \\ Lilia Aké, Universidad de Colima (México) \\ Silvia Etchegaray, Universidad Nacional de Río Cuarto (Argentina) \\ Aitzol Lasa, Universidad Pública de Navarra (España)
}

Basing on the onto-semiotic approach to mathematical knowledge and instruction a characterization of algebraic reasoning in primary education has been proposed in a previous paper, distinguishing three levels of algebraization. These levels were defined taking into account the types of representations used, the generalization processes involved and the analytical calculation at stakes in mathematical activity. In this paper we extend this previous model by including three additional advanced levels of algebraic reasoning that allow to analyses the mathematical activity carried out in secondary education. These new levels are based on the consideration of 1) use and processing of parameters to represent families of equations and functions; 2) the study of algebraic structures themselves, their definitions and properties. Furthermore, concordances and complementarities of this model with the three stages of algebrization proposed under the Anthropological Theory of Didactics (ATD) are analyzed.

The types of object and algebraic processes considered in the model described in this paper complement with microscopic analysis those that can be carried out using the ATD notion of praxeology. In fact, our model allows a detailed study of the subjects' behavior, which extends the institutional perspective achieved with the identification of mathematical and didactical praxeologies. Moreover, our proto-algebraic levels are more suited to stages where algebraic reasoning is incipient (third cycle of primary education and middle school education), while the successive stages of algebraization described in the works carried out under the ATD, are more focused on the characterization of algebra in higher educational levels. Thus, although the criteria used in both theoretical frameworks to define levels or stages in the process of algebraization are different, the theoretical analysis in both models are consistent and complementary; however, a deeper articulation of these models should be pursued in later studies

The algebraic reasoning levels described in this paper have implications for training, both primary and secondary school teachers. In addition to developing curricular proposals related to algebra from the earliest levels of education, the teacher need to act as the main agent of change in the introduction and development of algebraic reasoning in elementary classrooms, and its progression in secondary education. Reflecting on algebraic thinking objects and processes and being able to recognize them can help teachers identify the features of mathematical practices on which they can intervene to gradually increase the algebraization levels of students' mathematical activity. 

ontosemiótica y antropológica.

Additionally, considering the algebraization levels of mathematical activity can help reasoning awareness of gaps or discontinuities in didactical trajectories. These gaps involve the use of different registers of semiotic representation, their treatment and conversion, as well as the establishment of relations between conceptual, propositional, procedural and argumentative objects of higher generality 